\title{
Hydrodynamic modes of partially condensed Bose mixtures
}

\author{
J. Armaitis ${ }^{*}$ H.T.C. Stoof, and R.A. Duine \\ Institute for Theoretical Physics and Center for Extreme Matter and Emergent Phenomena, \\ Utrecht University, Leuvenlaan 4, 3584 CE Utrecht, The Netherlands
}

(Dated: June 23, 2021)

\begin{abstract}
We generalize the Landau-Khalatnikov hydrodynamic theory for superfluid helium to twocomponent (binary) Bose mixtures at arbitrary temperatures. In particular, we include the spin-drag terms that correspond to viscous coupling between the clouds. Therefore, our theory not only describes the usual collective modes of the individual components, e.g., first and second sound, but also results in new collective modes, where both constituents participate. We study these modes in detail and present their dispersions using thermodynamic quantities obtained within the Popov approximation.
\end{abstract}

PACS numbers: 03.75.Kk, 03.75.Mn, 05.30.Jp

\section{INTRODUCTION}

The realization of a Bose-Einstein condensate (BEC) in ultracold alkali-metal vapors [1 3 has ignited a rapid progress in the understanding of degenerate gases at low temperatures [4, 5. A large part of this understanding has been gained through the study of collective modes [6] 8. In particular, it has been shown that for a weakly interacting gas of bosons close to absolute zero temperature, the collective excitations are Bogoliubov quasiparticles 9 12] that are responsible for fascinating properties of the system, including superfluidity and quantum depletion of the condensate.

Work building upon the single-component ultracold gases has provided access to even richer systems. In particular, considering mixtures of several species of particles with the same (Bose-Bose or Fermi-Fermi mixtures [13, 14]) or different statistics (Bose-Fermi mixtures [15]) has become possible. These systems are known as binary mixtures or two-component gases. Arguably the simplest of them is a mixture of two different hyperfine states of the same bosonic atom. However, even this simple system poses important questions concerning the nature of its ground state and the excitations. Therefore, much work has been carried out on the static and dynamic properties of the two-component Bose gas [16 29], both in the uniform case and for the trapped case. Most of the effort has been concentrated on the zero-temperature physics, with only a few studies [30, 31] on the properties of binary Bose mixtures at nonzero temperature.

Having more than a single component in the gas also allows one to make a connection to the physics of spins, by introducing a pseudospin to distinguish the two components. In particular, one can consider ferromagnetic and antiferromagnetic states 3234 as well as spin dynamics 35, 36, and topological spin textures 32, 33, 3744. One kinetic effect concerning the spin dynamics is the so-called spin drag [45, 46]. This recently observed

\footnotetext{
* j.armaitis@uu.nl
}

47, 48 effect corresponds to the relaxation of the difference of the velocities between the two components. Understanding the interplay between the BEC, the thermal particles, and the spin degrees of freedom in this relatively simple and well-controlled two-component Bose gas might also offer some insights for interacting spinful degenerate systems of a rather different nature, such as the condensate of magnons 49, 50.

In this paper we build upon our previous results for the ferromagnetic Bose gas [42, but now consider a different situation where two condensates are present in the miscible regime. We tackle the problem of the collective modes of the two-component mixture both in the uniform gas and in a trap in an effort toward making a connection with experiments. The structure of the paper is as follows. In Sec. II we describe the Popov theory of the binary Bose mixture, and present relevant thermodynamic functions in that approximation, including the equation of state. We study the effects of spin drag in Sec. III. In Sec. IV we develop a linear hydrodynamic model that makes use of our previous thermodynamic results and describes a two-component system accounting for spin drag. We present the results for the uniform and trapped gas in Sec. V. Finally, we conclude in Sec. VI.

\section{MICROSCOPIC THEORY}

In this section we briefly describe the microscopic Popov theory of the two-component Bose mixture. The Popov theory is an extension of the Bogoliubov theory to relatively high temperatures, which includes an improved treatment of the excitations. Specifically, the Bogoliubov excitations are allowed to interact, and their interactions are treated in the Hartree-Fock approximation. Multi-component gases of bosons have been treated in the Bogoliubov framework before [51]. In particular, two-component mixtures have been considered in Refs. [21, 24, 52, and some results from the Popov theory have been presented in Ref. [31. The novelty of our results is twofold: We present the Popov analysis in the functional-integral formalism and calculate the thermo- 
dynamic properties of the balanced binary Bose gas. Our discussion on the Bogoliubov transformation follows the usual grand-canonical treatment of the problem. The single-component situation has been treated in this way in, for instance, Refs. [53, 54.

\section{A. General binary mixture}

In general, a grand-canonical partition function for two bosonic fields $\left(\phi_{\uparrow}\right.$ and $\left.\phi_{\downarrow}\right)$ that includes all the possible $s$-wave interactions is

$$
Z=\int d\left[\phi_{\uparrow}^{*}\right] d\left[\phi_{\uparrow}\right] d\left[\phi_{\downarrow}^{*}\right] d\left[\phi_{\downarrow}\right] e^{-S\left[\phi_{\uparrow}^{*}, \phi_{\uparrow}, \phi_{\downarrow}^{*}, \phi_{\downarrow}\right] / \hbar},
$$

where the action is

$$
\begin{aligned}
& S\left[\phi_{\uparrow}^{*}, \phi_{\uparrow}, \phi_{\downarrow}^{*}, \phi_{\downarrow}\right]=\int_{0}^{\hbar \beta} d \tau \int d \boldsymbol{x} \\
& \quad \times\left(\sum _ { \sigma = \uparrow , \downarrow } \left[\phi_{\sigma}^{*}\left(\hbar \partial_{\tau}-\frac{\hbar^{2} \nabla^{2}}{2 m_{\sigma}}-\mu_{\sigma}\right) \phi_{\sigma}\right.\right. \\
& \left.\left.\quad+\frac{1}{2} g_{\sigma \sigma} \phi_{\sigma}^{*} \phi_{\sigma}^{*} \phi_{\sigma} \phi_{\sigma}\right]+g_{\uparrow \downarrow} \phi_{\uparrow}^{*} \phi_{\downarrow}^{*} \phi_{\downarrow} \phi_{\uparrow}\right),
\end{aligned}
$$

and all the fields are considered at the position $\boldsymbol{x}$ and the imaginary time $\tau$. Moreover, $\beta=1 / k_{\mathrm{B}} T$ is the inverse thermal energy, $m_{\sigma}$ are the masses of the particles, and $g_{\sigma \sigma^{\prime}}$ are the two-body $T$ matrices describing the $s$-wave interactions.

From now on we focus on the symmetric case, where the masses are equal $m=m_{\uparrow}=m_{\downarrow}$, and the intraspecies interactions are the same and described by a single scattering length $a$. Thus,

$$
g=g_{\uparrow \uparrow}=g_{\downarrow \downarrow}=\frac{4 \pi \hbar^{2} a}{m} .
$$

The interspecies interactions are described by another scattering length $a_{\uparrow \downarrow}$, implying that

$$
g_{\uparrow \downarrow}=\frac{4 \pi \hbar^{2} a_{\uparrow \downarrow}}{m} .
$$

All the interactions are assumed to be repulsive, i.e., $g>$ 0 and $g_{\uparrow \downarrow}>0$. Furthermore, as opposed to our earlier work in Ref. 42, we here focus on the case with two separate condensates. In order for this to be possible, the condition

$$
g>g_{\uparrow \downarrow}
$$

has to be satisfied, as otherwise the two components demix [55.

We are now in a position to perform a fluctuation expansion for each species by putting

$$
\phi_{\sigma}(\boldsymbol{x}, \tau)=\phi_{0 \sigma}(\boldsymbol{x})+\phi_{\sigma}^{\prime}(\boldsymbol{x}, \tau),
$$

where the fluctuations $\phi_{\sigma}^{\prime}(\boldsymbol{x}, \tau)$ are, on average, zero. Moreover, the fluctuations are orthogonal to the condensate $\left\langle\phi_{\sigma}(\boldsymbol{x}, \tau)\right\rangle=\phi_{0 \sigma}(\boldsymbol{x})$ of the same species which means that

$$
\int d \boldsymbol{x}\left(\phi_{0 \sigma}^{*}(\boldsymbol{x}) \phi_{\sigma}^{\prime}(\boldsymbol{x}, \tau)+\phi_{0 \sigma}(\boldsymbol{x}) \phi_{\sigma}^{\prime *}(\boldsymbol{x}, \tau)\right)=0 .
$$

Since in what follows the relative phases of the condensates do not play a significant role, we choose both the condensate fields to be real,

$$
\phi_{0 \sigma}(\boldsymbol{x})=\sqrt{n_{0 \sigma}},
$$

where $n_{0 \sigma}$ is the atomic (number) density of the condensed $\sigma$ particles. Moreover, since we are considering the uniform case here, the condensate density has no spatial dependence. Expanding the fields in the action in this manner, we have for the action

$$
S=S_{0}+\sum_{\sigma=\uparrow, \downarrow} S_{1 \sigma}+S_{2}+S_{3}+S_{4},
$$

where the zeroth-order (Landau-free-energy) contribution is

$$
\begin{aligned}
S_{0}=\hbar \beta V( & -\mu_{\uparrow} n_{0 \uparrow}+g n_{0 \uparrow}^{2} / 2 \\
& \left.-\mu_{\downarrow} n_{0 \downarrow}+g n_{0 \downarrow}^{2} / 2+g_{\uparrow \downarrow} n_{0 \uparrow} n_{0 \downarrow}\right),
\end{aligned}
$$

the term linear in fluctuations reads

$$
\begin{array}{r}
S_{1 \sigma}\left[\phi_{\sigma}^{\prime *}, \phi_{\sigma}^{\prime}\right]=\int_{0}^{\hbar \beta} d \tau \int d \boldsymbol{x}\left[\phi _ { \sigma } ^ { \prime * } \left(-\mu_{\sigma}+g n_{0 \sigma}\right.\right. \\
\left.\left.+g_{\uparrow \downarrow} n_{0 \bar{\sigma}}\right) \sqrt{n_{0 \sigma}}+\text { c.c. }\right],
\end{array}
$$

and the quadratic term is

$$
\begin{aligned}
S_{2}\left[\phi_{\uparrow}^{\prime *}, \phi_{\uparrow}^{\prime}, \phi_{\downarrow}^{\prime *}, \phi_{\downarrow}^{\prime}\right] & =\int_{0}^{\hbar \beta} d \tau \int d \boldsymbol{x} \sum_{\sigma=\uparrow, \downarrow}\left[\phi_{\sigma}^{\prime *}\left(\hbar \partial_{\tau}-\frac{\hbar^{2} \nabla^{2}}{2 m}-\mu_{\sigma}+2 g n_{0 \sigma}+g_{\uparrow \downarrow} n_{0 \bar{\sigma}}\right) \phi_{\sigma}^{\prime}\right. \\
& \left.+\frac{g n_{0 \sigma}}{2}\left(\phi_{\sigma}^{\prime *} \phi_{\sigma}^{\prime *}+\phi_{\sigma}^{\prime} \phi_{\sigma}^{\prime}\right)\right]+g_{\uparrow \downarrow} \sqrt{n_{0 \uparrow} n_{0 \downarrow}} \int_{0}^{\hbar \beta} d \tau \int d \boldsymbol{x}\left(\phi_{\uparrow}^{\prime} \phi_{\downarrow}^{\prime}+\phi_{\uparrow}^{\prime *} \phi_{\downarrow}^{\prime}+\phi_{\uparrow}^{\prime} \phi_{\downarrow}^{\prime *}+\phi_{\uparrow}^{\prime *} \phi_{\downarrow}^{\prime *}\right),
\end{aligned}
$$

where all the fluctuation fields are evaluated at $(\boldsymbol{x}, \tau)$, and we have denoted the species opposite to $\sigma$ by $\bar{\sigma}$.
Furthermore, $S_{3}$ and $S_{4}$ terms describe the interactions between the fluctuations, 


$$
\begin{aligned}
S_{3}\left[\phi_{\uparrow}^{\prime *}, \phi_{\uparrow}^{\prime}, \phi_{\downarrow}^{\prime *}, \phi_{\downarrow}^{\prime}\right]=\int_{0}^{\hbar \beta} d \tau \int d \boldsymbol{x} & \left(\sum_{\sigma=\uparrow, \downarrow} g \sqrt{n_{0 \sigma}}\left(\phi_{\sigma}^{*} \phi_{\sigma}^{\prime *} \phi_{\sigma}^{\prime}+\phi_{\sigma}^{\prime} \phi_{\sigma}^{\prime} \phi_{\sigma}^{\prime *}\right)\right. \\
& \left.+g_{\uparrow \downarrow} \sqrt{n_{0 \uparrow}}\left(\phi_{\downarrow}^{\prime *} \phi_{\downarrow}^{\prime} \phi_{\uparrow}^{\prime}+\phi_{\downarrow}^{\prime} \phi_{\downarrow}^{\prime *} \phi_{\uparrow}^{\prime *}\right)+g_{\uparrow \downarrow} \sqrt{n_{0 \downarrow}}\left(\phi_{\uparrow}^{\prime *} \phi_{\downarrow}^{\prime} \phi_{\uparrow}^{\prime}+\phi_{\uparrow}^{\prime} \phi_{\downarrow}^{\prime *} \phi_{\uparrow}^{\prime *}\right)\right),
\end{aligned}
$$

and

$$
\begin{aligned}
& S_{4}\left[\phi_{\uparrow}^{\prime *}, \phi_{\uparrow}^{\prime}, \phi_{\downarrow}^{\prime *}, \phi_{\downarrow}^{\prime}\right]= \\
& \int_{0}^{\hbar \beta} d \tau \int d \boldsymbol{x}\left(\sum_{\sigma=\uparrow, \downarrow} \frac{g}{2} \phi_{\sigma}^{\prime *} \phi_{\sigma}^{\prime *} \phi_{\sigma}^{\prime} \phi_{\sigma}^{\prime}+g_{\uparrow \downarrow} \phi_{\uparrow}^{\prime *} \phi_{\downarrow}^{\prime *} \phi_{\downarrow}^{\prime} \phi_{\uparrow}^{\prime}\right) .
\end{aligned}
$$

Note that the terms $S_{3}$ and $S_{4}$ are neglected in the Bogoliubov theory.

We now perform the Hartree-Fock theory for the excitations, which involves the inclusion of the mean field,

$$
\left\langle\phi_{\sigma}^{* *} \phi_{\sigma}^{\prime}\right\rangle=n_{\sigma}^{\prime},
$$

where $n_{\sigma}^{\prime}$ is the density of the excitations of the $\sigma$ species such that the total density of a species is

$$
n_{\sigma}=n_{0 \sigma}+n_{\sigma}^{\prime} .
$$

Note that we neglect the coherence between the two species and take $\left\langle\phi_{\sigma}^{\prime *} \phi_{\bar{\sigma}}^{\prime}\right\rangle=0$. Therefore, the appropriate mean-field substitutions are

$$
\begin{array}{r}
\phi_{\sigma}^{\prime *} \phi_{\sigma}^{\prime *} \phi_{\sigma}^{\prime} \phi_{\sigma}^{\prime} \rightarrow 4 n_{\sigma}^{\prime} \phi_{\sigma}^{\prime *} \phi_{\sigma}^{\prime}-2 n_{\sigma}^{\prime 2}, \\
\phi_{\uparrow}^{\prime *} \phi_{\downarrow}^{\prime *} \phi_{\downarrow}^{\prime} \phi_{\uparrow}^{\prime} \rightarrow n_{\uparrow}^{\prime} \phi_{\downarrow}^{\prime *} \phi_{\downarrow}^{\prime}+n_{\downarrow}^{\prime} \phi_{\uparrow}^{\prime *} \phi_{\uparrow}^{\prime}-n_{\downarrow}^{\prime} n_{\uparrow}^{\prime},
\end{array}
$$

where the subtractions account for double counting in the quartic term of the action, whereas in the cubic terms no double-counting problems appear, as can be seen by applying Wick's theorem.

By requiring all the linear terms in $\phi_{\sigma}^{\prime}$ and $\phi_{\sigma}^{\prime *}$ of the action to vanish, we obtain a set of two Gross-Pitaevskii equations for the uniform condensates that read

$$
\begin{aligned}
& \left(-\mu_{\uparrow}+g n_{0 \uparrow}+g_{\uparrow \downarrow} n_{0 \downarrow}+2 g n_{\uparrow}^{\prime}+g_{\uparrow \downarrow} n_{\downarrow}^{\prime}\right) \sqrt{n_{0 \uparrow}}=0, \\
& \left(-\mu_{\downarrow}+g n_{0 \downarrow}+g_{\uparrow \downarrow} n_{0 \uparrow}+2 g n_{\downarrow}^{\prime}+g_{\uparrow \downarrow} n_{\uparrow}^{\prime}\right) \sqrt{n_{0 \downarrow}}=0,
\end{aligned}
$$

and from which the chemical potentials are obtained as

$$
\begin{aligned}
& \mu_{\uparrow}=g n_{0 \uparrow}+g_{\uparrow \downarrow} n_{0 \downarrow}+2 g n_{\uparrow}^{\prime}+g_{\uparrow \downarrow} n_{\downarrow}^{\prime}, \\
& \mu_{\downarrow}=g n_{0 \downarrow}+g_{\uparrow \downarrow} n_{0 \uparrow}+2 g n_{\downarrow}^{\prime}+g_{\uparrow \downarrow} n_{\uparrow}^{\prime} .
\end{aligned}
$$

In order to diagonalize the quadratic part of the action, we perform a Fourier transformation, and then introduce Nambu space [54]. Since we want to rewrite the quadratic part of the action in the form

$$
S_{2}\left[\phi_{\uparrow}^{\prime *}, \phi_{\uparrow}^{\prime}, \phi_{\downarrow}^{\prime *}, \phi_{\downarrow}^{\prime}\right]=-\frac{\hbar}{2} \sum_{\boldsymbol{k} \neq 0, n} \boldsymbol{\Phi}_{\boldsymbol{k} n} \cdot \boldsymbol{G}_{\boldsymbol{k} n}^{-1} \cdot \boldsymbol{\Phi}_{\boldsymbol{k} n}^{\dagger},
$$

where $\hbar \boldsymbol{k}$ is the momentum, $n$ labels the Matsubara frequencies $\omega_{n}=2 \pi n / \hbar \beta$,

$$
\boldsymbol{\Phi}_{\boldsymbol{k} n}=\left(\phi_{\uparrow \boldsymbol{k} n}^{* *}, \phi_{\uparrow-\boldsymbol{k} n}^{\prime}, \phi_{\downarrow k n}^{*}, \phi_{\downarrow-k n}^{\prime}\right),
$$

is a vector in the appropriate Nambu space in this case, and $\boldsymbol{G}^{-1}$ is the inverse Green's function of the system.
Note that we have to take care to preserve the correct time ordering. The latter results in an extra term in the action,

$$
\begin{aligned}
& S_{\mathrm{TO}}=-\frac{\hbar \beta}{2} \sum_{\sigma, \boldsymbol{k} \neq 0, n}\left[\varepsilon_{\boldsymbol{k}}-\mu_{\sigma}+g\left(2 n_{0 \sigma}+2 n_{\sigma}^{\prime}\right)\right. \\
& \left.+g_{\uparrow \downarrow}\left(n_{0 \bar{\sigma}}+n_{\bar{\sigma}}^{\prime}\right)\right]=-\frac{\hbar \beta}{2} \sum_{\sigma, \boldsymbol{k} \neq 0, n}\left(\varepsilon_{\boldsymbol{k}}+g n_{0 \sigma}\right),
\end{aligned}
$$

where $\varepsilon_{\boldsymbol{k}}=\hbar^{2} k^{2} / 2 m$ is the kinetic energy. Therefore, plugging the expressions for the chemical potentials $\mu_{\sigma}$ into the action, we have

$$
\begin{aligned}
S= & -\hbar \beta V\left(g_{\uparrow \downarrow} n_{\uparrow} n_{\downarrow}+g\left(n_{\downarrow}^{2}+n_{\uparrow}^{2}\right)\right. \\
& \left.-g \frac{n_{0 \downarrow}^{2}+n_{0 \uparrow}^{2}}{2}\right)-\frac{\hbar \beta}{2} \sum_{\sigma, \boldsymbol{k} \neq 0}\left(\varepsilon_{\boldsymbol{k}}+g n_{0 \sigma}\right) \\
& -\frac{\hbar}{2} \sum_{\boldsymbol{k} \neq 0, n} \boldsymbol{\Phi}_{\boldsymbol{k} n} \cdot \boldsymbol{G}_{\boldsymbol{k} n}^{-1} \cdot \boldsymbol{\Phi}_{\boldsymbol{k} n}^{\dagger},
\end{aligned}
$$

where $V$ is the volume of the system, while

$$
-\hbar \boldsymbol{G}_{\boldsymbol{k n}}^{-1}=\left(\begin{array}{cc}
-\hbar G_{\mathrm{B}, \uparrow \boldsymbol{k} n}^{-1} & \hbar \Sigma_{\uparrow \downarrow} \\
\hbar \Sigma_{\uparrow \downarrow} & -\hbar G_{\mathrm{B}, \downarrow \boldsymbol{k} n}^{-1}
\end{array}\right),
$$

where $G_{\mathrm{B}, \uparrow \boldsymbol{k} n}^{-1}$ and $\Sigma_{\uparrow \downarrow}$ are two-by-two submatrices (from now on two-by-two matrices are denoted by capital letters, while four-by-four matrices are denoted by bold capital letters). The submatrices on the diagonal are exactly the same as the Bogoliubov (single-component) inverse Green's functions, that is,

$$
-\hbar G_{\mathrm{B}, \sigma \boldsymbol{k} n}^{-1}=\left(\begin{array}{cc}
-i \hbar \omega_{n}+\varepsilon_{\boldsymbol{k}}+g n_{0 \sigma} & g n_{0 \sigma} \\
g n_{0 \sigma} & i \hbar \omega_{n}+\varepsilon_{\boldsymbol{k}}+g n_{0 \sigma}
\end{array}\right),
$$

and the off-diagonal matrix is the self-energy due to the interspecies coupling given by

$$
\hbar \Sigma_{\uparrow \downarrow}=g_{\uparrow \downarrow} \sqrt{n_{0 \uparrow} n_{0 \downarrow}}\left(\begin{array}{ll}
1 & 1 \\
1 & 1
\end{array}\right) .
$$

Anticipating the Bogoliubov transformation we define

$$
\boldsymbol{I}=\left(\begin{array}{cc}
\sigma_{z} & 0 \\
0 & \sigma_{z}
\end{array}\right)
$$

where

$$
\sigma_{z}=\left(\begin{array}{cc}
1 & 0 \\
0 & -1
\end{array}\right)
$$

Moreover, we define a matrix $\boldsymbol{\Gamma}_{\boldsymbol{k}}$ which is obtained by setting $\omega_{n}$ to zero in the inverse Green's function, i.e.,

$$
\Gamma_{\boldsymbol{k}}=-\hbar \boldsymbol{G}_{\boldsymbol{k} 0}^{-1} .
$$


We now proceed to diagonalize the matrix $\boldsymbol{\Gamma}_{\boldsymbol{k}}$ while keeping the bosonic character of the excitations, i.e., requiring that their operators obey bosonic commutation relations. In our formalism the preservation of the bosonic commutation relations is represented by the fact that the Matsubara-frequency terms are unaffected by the transformation. Hence, we look for real eigenvectors $\boldsymbol{w}$ of $\boldsymbol{I} \cdot \boldsymbol{\Gamma}_{\boldsymbol{k}}$

$$
\boldsymbol{I} \cdot \boldsymbol{\Gamma}_{\boldsymbol{k}} \cdot \boldsymbol{w}_{ \pm, \boldsymbol{k}}=E_{ \pm, \boldsymbol{k}} \boldsymbol{w}_{ \pm, \boldsymbol{k}}
$$

satisfying the property

$$
\boldsymbol{w}_{ \pm, \boldsymbol{k}} \cdot \boldsymbol{I} \cdot \boldsymbol{w}_{ \pm, \boldsymbol{k}}=1 \text {. }
$$

The above-mentioned eigenvalues define the dispersions of the Bogoliubov quasiparticles

$$
\begin{aligned}
E_{ \pm, \boldsymbol{k}}^{2}= & \varepsilon_{\boldsymbol{k}}\left(\varepsilon_{\boldsymbol{k}}+g\left(n_{0 \uparrow}+n_{0 \downarrow}\right)\right) \\
& \pm \varepsilon_{\boldsymbol{k}} \sqrt{g^{2}\left(n_{0 \uparrow}-n_{0 \downarrow}\right)^{2}+4 g_{\uparrow \downarrow}^{2} n_{0 \uparrow} n_{0 \downarrow}} .
\end{aligned}
$$

The eigenvectors $\boldsymbol{w}_{ \pm, \boldsymbol{k}}$ have the so-called Bogoliubov coherence factors as their entries, or explicitly

$$
\boldsymbol{w}_{ \pm, \boldsymbol{k}}=\left(u_{ \pm, \boldsymbol{k}}^{\uparrow},-v_{ \pm, \boldsymbol{k}}^{\uparrow}, u_{ \pm, \boldsymbol{k}}^{\downarrow},-v_{ \pm, \boldsymbol{k}}^{\downarrow}\right) .
$$

For the case at hand these coherence factors are rather involved functions of the momentum. Since they have been presented explicitly in Ref. [24], we do not write them out here.

The relation between the two original fluctuation fields and the new Bogoliubov quasiparticle fields is given by

$$
\boldsymbol{\Phi}_{\boldsymbol{k} n}=\boldsymbol{W}_{\boldsymbol{k}} \cdot \boldsymbol{\Psi}_{\boldsymbol{k} n},
$$

where we have defined the Bogoliubov fields

$$
\boldsymbol{\Psi}_{\boldsymbol{k} n}=\left(\psi_{+, \boldsymbol{k} n}^{*}, \psi_{+,-\boldsymbol{k} n}, \psi_{-, \boldsymbol{k} n}^{*}, \psi_{-,-\boldsymbol{k} n}\right),
$$

and the transformation matrix

$$
\boldsymbol{W}_{\boldsymbol{k}}=\left(\begin{array}{cc}
W_{+, \boldsymbol{k}}^{\uparrow} & W_{-, \boldsymbol{k}}^{\uparrow} \\
W_{+, \boldsymbol{k}}^{\downarrow} & W_{-, \boldsymbol{k}}^{\downarrow}
\end{array}\right)
$$

which consists of the submatrices

$$
W_{s, \boldsymbol{k}}^{\sigma}=\left(\begin{array}{cc}
u_{s, \boldsymbol{k}}^{\sigma} & -v_{s, \boldsymbol{k}}^{\sigma} \\
-v_{s, \boldsymbol{k}}^{\sigma} & u_{s, \boldsymbol{k}}^{\sigma}
\end{array}\right) .
$$

It is straightforward to check that this transformation leaves the Matsubara-frequency terms in the action unaffected, and therefore the Bogoliubov excitations are bosons. The action becomes

$$
\begin{aligned}
S= & -\hbar \beta V\left(g_{\uparrow \downarrow} n_{\uparrow} n_{\downarrow}+g\left(n_{\downarrow}^{2}+n_{\uparrow}^{2}\right)-g \frac{n_{0 \downarrow}^{2}+n_{0 \uparrow}^{2}}{2}\right) \\
& +\frac{\hbar \beta}{2} \sum_{\boldsymbol{k} \neq 0}\left(E_{+, \boldsymbol{k}}+E_{-, \boldsymbol{k}}-2 \varepsilon_{\boldsymbol{k}}-g\left(n_{0 \uparrow}+n_{0 \downarrow}\right)\right) \\
& +\sum_{s= \pm} \sum_{\boldsymbol{k} \neq 0, n}\left(-i \hbar \omega_{n}+E_{s, \boldsymbol{k}}\right) \psi_{s, \boldsymbol{k} n}^{*}, \psi_{s, \boldsymbol{k} n}
\end{aligned}
$$

where $\left(E_{+, \boldsymbol{k}}+E_{-, \boldsymbol{k}}\right)$ in the second term is again due to the time ordering but this time of the $\psi_{ \pm}$fields.

In the preceding equation, the first term describes the Hartree-Fock contribution to the action. The second term (after properly accounting for the fact that the contact potential does not fall off at high momenta) can be shown to describe the so-called Lee-Huang-Yang correction [56, 57, which is small for a weakly interacting gas, and can therefore be safely neglected. The last term describes the Bogoliubov excitations. Evaluating this path integral amounts to a Gaussian integration and can be performed exactly. Finally, we perform the remaining bosonic Matsubara sum 54

$$
\begin{array}{r}
\lim _{\eta \rightarrow 0^{+}} \sum_{n} \ln \left[\beta\left(-i \hbar \omega_{n}+E_{s, \boldsymbol{k}}\right)\right] e^{i \omega_{n} \eta} \\
=\ln \left(1-e^{-\beta E_{s, \boldsymbol{k}}}\right),
\end{array}
$$

and arrive at the following expression for the partition function

$$
\begin{array}{r}
Z=\exp \left[\beta\left(g_{\uparrow \downarrow} n_{\uparrow} n_{\downarrow}+g\left(n_{\downarrow}^{2}+n_{\uparrow}^{2}\right)-g \frac{n_{0 \downarrow}^{2}+n_{0 \uparrow}^{2}}{2}\right)\right. \\
\left.-\frac{1}{V} \sum_{s= \pm, \boldsymbol{k} \neq 0} \ln \left(1-e^{-\beta E_{s, \boldsymbol{k}}}\right)\right] .
\end{array}
$$

The partition function is related to the pressure by

$$
\begin{aligned}
p\left(n_{0 \uparrow}, n_{0 \downarrow}, T\right)= & -\Omega / V=\frac{1}{\beta} \ln (Z) \\
= & g_{\uparrow \downarrow} n_{\uparrow} n_{\downarrow}+g\left(n_{\downarrow}^{2}+n_{\uparrow}^{2}\right)-g \frac{n_{0 \downarrow}^{2}+n_{0 \uparrow}^{2}}{2} \\
& -\frac{1}{\beta V} \sum_{s= \pm, \boldsymbol{k} \neq 0} \ln \left(1-e^{-\beta E_{s, \boldsymbol{k}}}\right),
\end{aligned}
$$

where $\Omega$ is the grand potential. We have obtained the (average) particle densities

$$
n_{\sigma}=n_{\sigma 0}+\frac{1}{V} \sum_{s= \pm, \boldsymbol{k} \neq 0} \frac{\left|u_{s, \boldsymbol{k}}^{\sigma}\right|^{2}+\left|v_{s, \boldsymbol{k}}^{\sigma}\right|^{2}}{e^{\beta E_{s}}-1}
$$

from the appropriate Green's functions, while the entropy per volume 58, is

$$
\begin{aligned}
\frac{S}{V}=-\left.\frac{\partial \Omega}{\partial T}\right|_{n_{\sigma}^{\prime}, n_{0 \sigma}}= & -\frac{k_{B}}{V} \sum_{s= \pm, \boldsymbol{k} \neq 0} \ln \left(1-e^{-\beta E_{s, \boldsymbol{k}}}\right) \\
& +\frac{k_{B}}{V} \sum_{s= \pm, \boldsymbol{k} \neq 0} \frac{\beta E_{s, \boldsymbol{k}}}{e^{\beta E_{s, \boldsymbol{k}}-1}} .
\end{aligned}
$$

For future convenience, we define the entropy per particle as

$$
s \equiv \frac{S}{V} \frac{1}{n_{\uparrow}+n_{\downarrow}}
$$

and also the total chemical potential $\mu_{\text {tot }}$, as well as the difference of the chemical potentials

$$
\begin{aligned}
\mu_{\text {tot }} & =\mu_{\uparrow}+\mu_{\downarrow}, \\
\Delta \mu & =\mu_{\uparrow}-\mu_{\downarrow} .
\end{aligned}
$$

Similarly, we define the total particle density and the difference of the particle densities:

$$
\begin{aligned}
n_{\text {tot }} & =n_{\uparrow}+n_{\downarrow}, \\
\Delta n & =n_{\uparrow}-n_{\downarrow} .
\end{aligned}
$$

Finally, it is also beneficial to define two additional $T$ 
matrices,

$$
g_{ \pm}=g \pm g_{\uparrow \downarrow}=g(1 \pm \gamma),
$$

where we have also introduced a dimensionless number $\gamma$, which shows the relative strength between the interspecies and the intraspecies repulsion. Note that in the miscible case that we discuss here, $\gamma<1$.

\section{B. Balanced mixture}

Of particular interest is the balanced case, where the number of particles of the two species are equal: $n_{\uparrow}=$ $n_{\downarrow}=n$. We consider it in this subsection. An obvious consequence of this limit is

$$
n_{0 \uparrow}=n_{0 \downarrow} \equiv n_{0},
$$

and

$$
\mu \equiv \mu_{\uparrow}=\mu_{\downarrow}=g_{+} n+g n^{\prime} .
$$

Moreover, the dispersions of the quasiparticles become

$$
E_{ \pm, \boldsymbol{k}}^{2}=\varepsilon_{\boldsymbol{k}}\left(\varepsilon_{\boldsymbol{k}}+2 n_{0} g_{ \pm}\right)
$$

and the Bogoliubov transformation matrix simplifies considerably to

$$
\boldsymbol{W}_{\boldsymbol{k}}=\left(\begin{array}{ll}
W_{+, \boldsymbol{k}} & W_{-, \boldsymbol{k}} \\
W_{+, \boldsymbol{k}} & W_{-, \boldsymbol{k}}
\end{array}\right),
$$

which can be inverted to

$$
\boldsymbol{W}_{\boldsymbol{k}}^{-1}=\frac{1}{2}\left(\begin{array}{cc}
W_{+, \boldsymbol{k}}^{-1} & W_{+, \boldsymbol{k}}^{-1} \\
-W_{-, \boldsymbol{k}}^{-1} & W_{-, \boldsymbol{k}}^{-1}
\end{array}\right)
$$

from which we can conclude that the $\psi_{+, \text {, } n}$ field has equal contributions from the $\phi_{\uparrow k n}$ and $\phi_{\downarrow k n}$ fields, whereas the latter fields enter $\psi_{-, k n}$ with a relative minus sign but with equal absolute weights. This implies that $\psi_{+, k n}$ describes densitylike excitations, whereas $\psi_{-, \boldsymbol{k}}$ describes spinlike excitations. The submatrices $W_{s, \boldsymbol{k}}$ in the above are

$$
W_{s, \boldsymbol{k}}=\left(\begin{array}{cc}
u_{s, \boldsymbol{k}} & -v_{s, \boldsymbol{k}} \\
-v_{s, \boldsymbol{k}} & u_{s, \boldsymbol{k}}
\end{array}\right)
$$

where in this case the coherence factors are simple enough to be written out explicitly as

$$
\begin{aligned}
& v_{ \pm, \boldsymbol{k}}^{2}=\frac{1}{4}\left(\frac{\varepsilon_{p}+g_{ \pm} n_{0}}{E_{ \pm, \boldsymbol{k}}}-1\right), \\
& u_{ \pm, \boldsymbol{k}}^{2}=\frac{1}{4}\left(\frac{\varepsilon_{p}+g_{ \pm} n_{0}}{E_{ \pm, \boldsymbol{k}}}+1\right) .
\end{aligned}
$$

Note that the coherence factors are very similar to the single-species case. However, the prefactor $(1 / 4)$ here is different from the single-species case $(1 / 2)$, since the transformation now involves four fields instead of two.

We now proceed to discuss the thermodynamic functions of the balanced binary mixture. Throughout the discussion, we consider three different dimensionless interaction parameters $n^{1 / 3} a=0.01,0.05$, and 0.1. They have been chosen to correspond to the experimentally relevant weakly interacting (far away from Feshbach resonances) ultracold gas situations. In particular, we consider the sodium atom which has several scattering lengths between the accessible hyperfine levels close to $50 \mathrm{Bohr}$ radii [59]. Moreover, we are interested in the hydrodynamic regime, where the density in the center of the trap might become as high as $10^{21} \mathrm{~m}^{-3}$ [60], which corresponds to $n^{1 / 3} a \simeq 0.03$. Comparing thermodynamic quantities calculated within the Bogoliubov theory with the renormalization group results for the single-species case (see Ref. 61]) shows that the two agree very well all the way up to the condensation temperature $T_{c}$ for $n^{1 / 3} a=0.01$. Agreement for $n^{1 / 3} a=0.05$ and particularly for 0.1 is less good. However, the qualitative features of the thermodynamic functions are preserved. We expect the situation to be similar for the two-species case.

We present the equation of state (pressure) in Fig. 1. We scale the pressure plots with the zero-temperature pressure $g_{+} n^{2} / 2$ obtained from a Gross-Pitaevskii calculation [55]. For increasing interaction strength, the scaled pressure decreases, since the thermal energy becomes comparable with the interaction energy at higher temperature. For $\gamma=0$, the pressure is equal to the pressure of a single species of gas with twice the density (see, e.g., Ref. 61] for comparison).

Note that for a fixed total number of particles, the pressure is not a monotonically increasing function of temperature in the Bogoliubov theory, as opposed to the Popov theory. This spurious effect appears due to the competition between the depletion of the condensate (decreases pressure) and the population of the thermal states (increases pressure). Moreover, this leads to a spurious lack of avoided crossing of the first- and second-sound velocities, therefore necessitating the use of at least the Popov theory to describe the sound velocities accurately.

The entropy per particle is presented in Fig. 2, Since the system is described by a mixture of noninteracting phonon gases at low temperatures, the entropy per particle shows a $T^{3}$ power-law behavior at low temperatures. Furthermore, the phonon velocity scales with the interaction strength and therefore stronger-interacting gases have a higher entropy.

\section{SPIN DRAG IN A PARTIALLY-CONDENSED BOSE GAS}

In this section we investigate kinetic (incoherent) processes that contribute to density and velocity dynamics for the balanced binary Bose gas below the critical temperature. We start by generalizing the results of the kinetic theory for a uniform single-species Bose gas (see, for instance, Ref. 62]) to the two-species gas. The collision integrals that we consider below can be formally derived from the Heisenberg equations of motion for the atoms of the two different species. For a detailed discussion of that derivation for the case of a single species, we refer to 

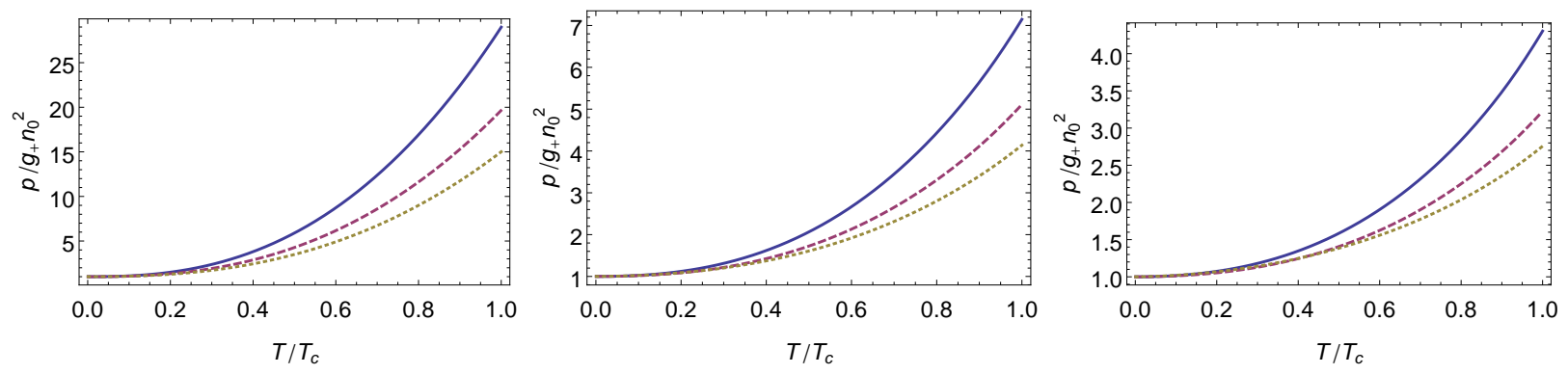

FIG. 1. (Color online) Pressure of the two-species balanced gas. The interaction parameter increases from left to right: $n^{1 / 3} a=0.01,0.05$, and 0.1 . Intercomponent interactions are zero $(\gamma=0)$ for the solid line, moderate $(\gamma=1 / 2)$ for the dashed line, and strong $(\gamma \lesssim 1)$ for the dotted line.
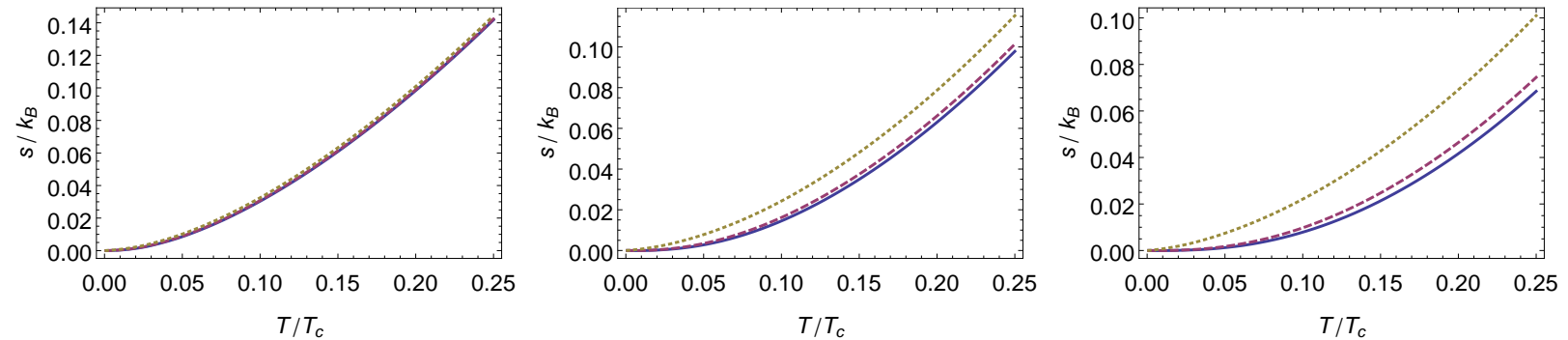

FIG. 2. (Color online) The entropy per particle of the two-species balanced gas. The interaction parameter increases from left to right: $n^{1 / 3} a=0.01,0.05$, and 0.1. Intercomponent interactions are zero $(\gamma=0)$ for the solid line, moderate $(\gamma=1 / 2)$ for the dashed line, and strong $(\gamma \lesssim 1)$ for the dotted line. A limited temperature range is depicted to emphasize the difference between the curves.

Ref. 63. The collision integrals discussed in this section conserve the number of $\uparrow$ and $\downarrow$ particles separately.

Since the kinetic processes in question vanish for very low temperatures, and in order to avoid complications posed by the fact that the Bogoliubov transformation mixes the spin species, we work in the Hartree-Fock approximation in this section. The Hartree-Fock approximation is therefore valid for the whole range of temperatures where the collision processes play a significant role. Moreover, here we consider the "local equilibrium" situation where the chemical potentials of the thermal atoms and the condensates are equal to $\mu=g_{+} n+g n^{\prime}$. The thermal atoms thus feel the Hartree-Fock mean-field energy $\left(g_{+}+g\right) n$. These considerations lead to a BoseEinstein distribution with a nonzero average momentum $\boldsymbol{p}_{\mathrm{nc} \uparrow}\left(\boldsymbol{p}_{\mathrm{nc} \downarrow}\right)$ for the noncondensed $\uparrow(\downarrow)$ atoms,

$$
f_{i}^{\alpha}=\frac{1}{e^{\beta\left(\boldsymbol{p}_{i}-\boldsymbol{p}_{\mathrm{nc} \sigma}\right)^{2} / 2 m+\beta\left(g_{+}+g\right) n-\beta \mu}-1},
$$

where $\sigma$ labels the spin species and $i$ labels the momentum variable $\boldsymbol{p}_{i}$ (the omission of the $i$ label denotes the momentum variable $\boldsymbol{p}$ ). Furthermore, we allow for different nonzero momenta of the two condensates: The $\uparrow$ $(\downarrow)$ condensate has a momentum of $\boldsymbol{p}_{\mathrm{c} \uparrow}\left(\boldsymbol{p}_{\mathrm{c} \downarrow}\right)$.

In the single-species case only one collision process, namely $C_{12}$, using the notation of Ref. [63], is responsible for the relaxation of the difference between the condensate velocity and the velocity of the thermal atoms. However, five different processes (see Fig. 3) can con- tribute to the dynamics of various densities and velocities in the two-species system, leading to the following set of quantum kinetic equations for the distributions of the thermal particles, where we only consider the collisional contributions:

$$
\begin{aligned}
& \left.\partial_{t} f^{\uparrow}\right|_{\text {coll }}=C_{22}^{\uparrow \downarrow}+C_{12}^{\uparrow \uparrow}+C_{12}^{\uparrow \downarrow}+\bar{C}_{12}^{\downarrow \uparrow}, \\
& \left.\partial_{t} f^{\downarrow}\right|_{\text {coll }}=\bar{C}_{22}^{\uparrow \downarrow}+C_{12}^{\downarrow \downarrow}+\bar{C}_{12}^{\uparrow \downarrow}+C_{12}^{\downarrow \uparrow} .
\end{aligned}
$$

We now discuss the individual collision terms present in these equations. We use a shorthand notation for the momentum integrals $\int_{i} \equiv \int d^{3} \boldsymbol{p}_{i}$ in order to simplify the following formulas. First, we have a spin-drag term (Fig. 3 (a); cf. Refs. [46, 48), which only involves scattering between the thermal atoms and thus exists both below and above the critical temperature,

$$
\begin{aligned}
& C_{22}^{\uparrow \downarrow}=\int_{1234} A_{22}\left[\delta^{(3)}\left(\boldsymbol{p}-\boldsymbol{p}_{4}\right)-\delta^{(3)}\left(\boldsymbol{p}-\boldsymbol{p}_{1}\right)\right], \\
& \bar{C}_{22}^{\uparrow \downarrow}=\int_{1234} A_{22}\left[\delta^{(3)}\left(\boldsymbol{p}-\boldsymbol{p}_{3}\right)-\delta^{(3)}\left(\boldsymbol{p}-\boldsymbol{p}_{2}\right)\right],
\end{aligned}
$$

where we have denoted the common part of the integrand by

$$
\begin{aligned}
& A_{22}= \frac{g_{\uparrow \downarrow}^{2}}{(2 \pi)^{5} \hbar^{7}} \delta^{(3)}\left(\boldsymbol{p}_{1}+\boldsymbol{p}_{2}-\boldsymbol{p}_{3}-\boldsymbol{p}_{4}\right) \\
& \times \delta\left(\left[\boldsymbol{p}_{1}^{2}+\boldsymbol{p}_{2}^{2}-\boldsymbol{p}_{3}^{2}-\boldsymbol{p}_{4}^{2}\right] / 2 m\right) \\
& \times\left[f_{1}^{\uparrow} f_{2}^{\downarrow}\left(1+f_{3}^{\downarrow}\right)\left(1+f_{4}^{\uparrow}\right)-\left(1+f_{1}^{\uparrow}\right)\left(1+f_{2}^{\downarrow}\right) f_{3}^{\downarrow} f_{4}^{\uparrow}\right] .
\end{aligned}
$$




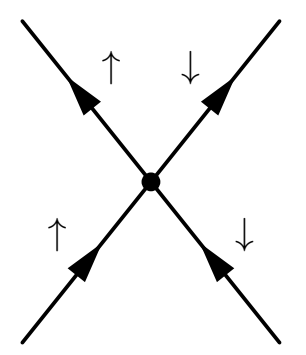

(a) $C_{22}^{\uparrow \downarrow}$

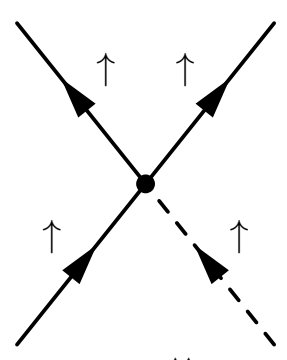

(b) $C_{12}^{\uparrow \uparrow}$

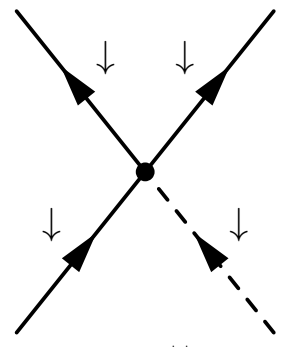

(c) $C_{12}^{\downarrow \downarrow}$



(d) $C_{12}^{\uparrow \downarrow}$

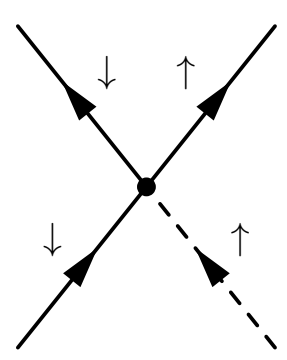

(e) $C_{12}^{\perp \uparrow}$

FIG. 3. Diagrams corresponding to the relevant collision integrals. Solid lines depict thermal atoms, while dashed lines represent condensed atoms.

This term contributes to the relaxation of the difference of momenta $\boldsymbol{p}_{\text {nc } \uparrow}-\boldsymbol{p}_{\text {nc } \downarrow}$. Furthermore, we consider the following intraspecies collision terms [Figs. 3(b) and[3(c)], which explicitly depend on the condensate density and thus only exist below the critical temperature,

$$
\begin{aligned}
C_{12}^{\uparrow \uparrow(\downarrow \downarrow)}= & \int_{123} A_{12}^{\uparrow \uparrow(\downarrow \downarrow)}\left[\delta^{(3)}\left(\boldsymbol{p}-\boldsymbol{p}_{1}\right)\right. \\
& \left.-\delta^{(3)}\left(\boldsymbol{p}-\boldsymbol{p}_{2}\right)-\delta^{(3)}\left(\boldsymbol{p}-\boldsymbol{p}_{3}\right)\right],
\end{aligned}
$$

where

$$
\begin{aligned}
& A_{12}^{\uparrow \uparrow(\downarrow \downarrow)}=\frac{2 g^{2} n_{0 \uparrow(\downarrow)}}{(2 \pi)^{2} \hbar^{4}} \delta^{(3)}\left(\boldsymbol{p}_{1}+\boldsymbol{p}_{\mathrm{c} \uparrow(\downarrow)}-\boldsymbol{p}_{2}-\boldsymbol{p}_{3}\right) \\
& \quad \times \delta\left(\left[\boldsymbol{p}_{1}^{2}+\boldsymbol{p}_{\mathrm{c} \uparrow(\downarrow)}^{2}-\boldsymbol{p}_{2}^{2}-\boldsymbol{p}_{3}^{2}\right] / 2 m-g n_{0 \uparrow(\downarrow)}\right) \\
& \quad \times\left[f_{1}^{\uparrow(\downarrow)}\left(1+f_{2}^{\uparrow(\downarrow)}\right)\left(1+f_{3}^{\uparrow(\downarrow)}\right)-\left(1+f_{1}^{\uparrow(\downarrow)}\right) f_{2}^{\uparrow(\downarrow)} f_{3}^{\uparrow(\downarrow)}\right] .
\end{aligned}
$$

These terms contribute to the dynamics of the condensate fraction and also describe the relaxation between the condensate velocity and the average thermal particle velocity of the same species. Moreover, we also have similar terms for the interspecies scattering, namely,

$$
\begin{aligned}
& C_{12}^{\uparrow \downarrow(\downarrow \uparrow)}=\int_{123} A_{12}^{\uparrow \downarrow(\downarrow \uparrow)}\left[\delta^{(3)}\left(\boldsymbol{p}-\boldsymbol{p}_{2}\right)-\delta^{(3)}\left(\boldsymbol{p}-\boldsymbol{p}_{1}\right)\right] \\
& \bar{C}_{12}^{\uparrow \downarrow(\downarrow \uparrow)}=\int_{123} A_{12}^{\uparrow \downarrow(\downarrow \uparrow)} \delta^{(3)}\left(\boldsymbol{p}-\boldsymbol{p}_{3}\right),
\end{aligned}
$$

where

$$
\begin{aligned}
& A_{12}^{\uparrow \downarrow(\uparrow \downarrow)}=\frac{g_{\uparrow \downarrow}^{2} n_{0 \downarrow}(\uparrow)}{(2 \pi)^{2} \hbar^{4}} \delta^{(3)}\left(\boldsymbol{p}_{1}+\boldsymbol{p}_{\mathrm{c} \downarrow(\uparrow)}-\boldsymbol{p}_{2}-\boldsymbol{p}_{3}\right) \\
& \times \delta\left(\left[\boldsymbol{p}_{1}^{2}+\boldsymbol{p}_{\mathrm{c} \downarrow(\uparrow)}^{2}-\boldsymbol{p}_{2}^{2}-\boldsymbol{p}_{3}^{2}\right] / 2 m-g n_{0 \downarrow(\uparrow)}\right) \\
& \times\left[f_{1}^{\uparrow(\downarrow)}\left(1+f_{2}^{\uparrow(\downarrow)}\right)\left(1+f_{3}^{\downarrow(\uparrow)}\right)-\left(1+f_{1}^{\uparrow(\downarrow)}\right) f_{2}^{\uparrow(\downarrow)} f_{3}^{\downarrow(\uparrow)}\right] .
\end{aligned}
$$

The latter terms also describe the dynamics of the condensate fraction, as well as the relaxation of various velocities mediated by the condensate.

In order to obtain the equations for the change of the density and the momentum of the thermal particles, we perform an integration of Eqs. (62) and $\sqrt{63}$ leading to

$$
\begin{array}{r}
\partial_{t} n_{\uparrow(\downarrow)}^{\prime}=\int \frac{d^{3} \boldsymbol{p}}{(2 \pi \hbar)^{3}} \partial_{t} f_{\uparrow(\downarrow)}, \\
\partial_{t}\left(n_{\uparrow(\downarrow)}^{\prime} \boldsymbol{p}_{\mathrm{nc} \uparrow(\downarrow)}\right)=\int \frac{d^{3} \boldsymbol{p}}{(2 \pi \hbar)^{3}} \boldsymbol{p} \partial_{t} f_{\uparrow(\downarrow)} .
\end{array}
$$

Adding the equations that change the thermal densities and the equations that change the condensate densities obtained from straightforward considerations of the collision integrals, we have

$$
\begin{aligned}
\partial_{t} n_{\uparrow}^{\prime} & =\Gamma_{12}^{\uparrow \uparrow}+\bar{\Gamma}_{12}^{\downarrow \uparrow}, \\
\partial_{t} n_{\downarrow}^{\prime} & =\Gamma_{12}^{\downarrow \downarrow}+\bar{\Gamma}_{12}^{\uparrow \downarrow}, \\
\partial_{t} n_{0 \uparrow} & =-\Gamma_{12}^{\uparrow \uparrow}-\bar{\Gamma}_{12}^{\downarrow \uparrow}, \\
\partial_{t} n_{0 \downarrow} & =-\Gamma_{12}^{\downarrow \downarrow}-\bar{\Gamma}_{12}^{\uparrow \downarrow},
\end{aligned}
$$

where

$$
\begin{aligned}
& \Gamma_{12}^{\uparrow \uparrow(\downarrow \downarrow)}=-\frac{1}{(2 \pi \hbar)^{3}} \int_{123} A_{12}^{\uparrow \uparrow(\downarrow \downarrow)}, \\
& \bar{\Gamma}_{12}^{\uparrow \downarrow(\downarrow \uparrow)}=\frac{1}{(2 \pi \hbar)^{3}} \int_{123} A_{12}^{\uparrow \downarrow(\uparrow \downarrow)},
\end{aligned}
$$

and we see that the total densities $n_{\uparrow(\downarrow)}$ are conserved separately. In a similar manner, the equations for the change of momenta are

$$
\begin{aligned}
& \partial_{t}\left(n_{\uparrow}^{\prime} \boldsymbol{p}_{\mathrm{nc} \uparrow}\right)=\frac{1}{(2 \pi \hbar)^{3}} \int_{1234}\left(\boldsymbol{p}_{4}-\boldsymbol{p}_{1}\right) A_{22}-\boldsymbol{p}_{\mathrm{c} \uparrow} \Gamma_{12}^{\uparrow \uparrow} \\
& +\frac{1}{(2 \pi \hbar)^{3}} \int_{123}\left(\boldsymbol{p}_{2}-\boldsymbol{p}_{1}\right) A_{12}^{\uparrow \downarrow}+\frac{1}{(2 \pi \hbar)^{3}} \int_{123} \boldsymbol{p}_{3} A_{12}^{\downarrow \uparrow}, \\
& \partial_{t}\left(n_{\downarrow}^{\prime} \boldsymbol{p}_{\mathrm{nc} \downarrow}\right)=-\frac{1}{(2 \pi \hbar)^{3}} \int_{1234}\left(\boldsymbol{p}_{4}-\boldsymbol{p}_{1}\right) A_{22}-\boldsymbol{p}_{\mathrm{c} \downarrow} \Gamma_{12}^{\downarrow \downarrow} \\
& +\frac{1}{(2 \pi \hbar)^{3}} \int_{123} \boldsymbol{p}_{3} A_{12}^{\uparrow \downarrow}+\frac{1}{(2 \pi \hbar)^{3}} \int_{123}\left(\boldsymbol{p}_{2}-\boldsymbol{p}_{1}\right) A_{12}^{\downarrow \uparrow}, \\
& \partial_{t}\left(n_{0 \uparrow} \boldsymbol{p}_{\mathrm{c} \uparrow}\right)=\boldsymbol{p}_{\mathrm{c} \uparrow} \Gamma_{12}^{\uparrow \uparrow}+\boldsymbol{p}_{\mathrm{c} \uparrow} \Gamma_{12}^{\downarrow \uparrow}, \\
& \partial_{t}\left(n_{0 \downarrow} \boldsymbol{p}_{\mathrm{c} \downarrow}\right)=\boldsymbol{p}_{\mathrm{c} \downarrow} \Gamma_{12}^{\downarrow \downarrow}+\boldsymbol{p}_{\mathrm{c} \downarrow} \Gamma_{12}^{\uparrow \downarrow} .
\end{aligned}
$$

Note that the total momentum is conserved; thus,

$$
\partial_{t}\left(n_{\uparrow}^{\prime} \boldsymbol{p}_{\mathrm{nc} \uparrow}+n_{\downarrow}^{\prime} \boldsymbol{p}_{\mathrm{nc} \downarrow}+n_{0 \uparrow} \boldsymbol{p}_{\mathrm{c} \uparrow}+n_{0 \downarrow} \boldsymbol{p}_{\mathrm{c} \downarrow}\right)=0 .
$$

For the hydrodynamic theory in the subsequent section, we are interested in the linearization of the collision integrals in terms of the velocity differences. It is 
straightforward to show that all the $\Gamma_{12}^{\alpha \beta}$ integrals are at least quadratic in terms of the momenta and, therefore, in linear response the densities stay constant for both species and $\partial_{t} n_{\alpha}^{\prime}=\partial_{t} n_{0 \alpha}=0$. In addition, this result implies that the condensate momentum experiences no linear relaxation. This is consistent with the common physical intuition that the condensate motion should not decay in the lowest order, as the condensate motion corresponds to the flow of a superfluid.

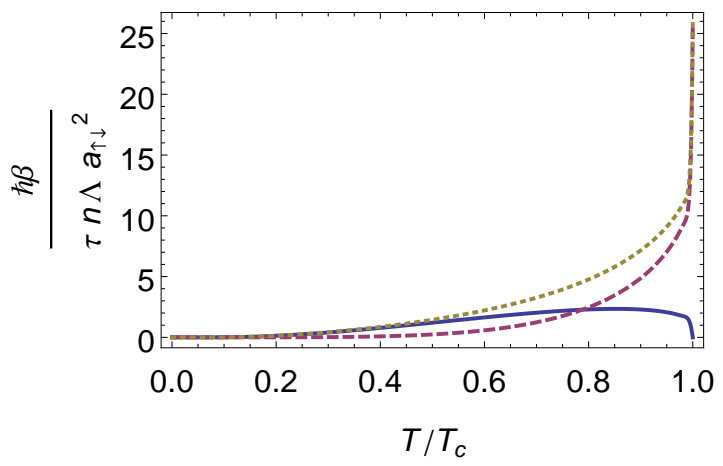

FIG. 4. (Color online) Condensate-assisted (solid line), thermal (dashed line), and total (dotted line) spin-drag rates for a system of sodium atoms at a density of $10^{21} \mathrm{~m}^{-3}, \gamma \simeq 1$, $n^{1 / 3} a=0.03$.

Nevertheless, the momentum difference between the two species of thermal particles experiences relaxation,

$$
\begin{aligned}
\partial_{t}\left(\boldsymbol{p}_{\mathrm{nc} \uparrow}-\boldsymbol{p}_{\mathrm{nc} \downarrow}\right) & =-\left(\frac{1}{\tau_{22}}+\frac{1}{\tau_{12}}\right)\left(\boldsymbol{p}_{\mathrm{nc} \uparrow}-\boldsymbol{p}_{\mathrm{nc} \downarrow}\right) \\
& =-\frac{1}{\tau_{\mathrm{sd}}}\left(\boldsymbol{p}_{\mathrm{nc} \uparrow}-\boldsymbol{p}_{\mathrm{nc} \downarrow}\right),
\end{aligned}
$$

where we have also defined the total spin-drag rate $1 / \tau_{\text {sd }}$ in terms of the thermal and condensate-assisted spin-drag relaxation rates $1 / \tau_{22}$ and $1 / \tau_{12}$, respectively. While the thermal spin-drag relaxation time

$$
\begin{gathered}
\frac{\hbar \beta}{\tau_{22} n \Lambda a_{\uparrow \downarrow}^{2}}=\frac{1}{6 \pi^{2}} \frac{1}{\left(n \Lambda^{3}\right)^{2}} \int_{0}^{\infty} \frac{d q d \omega q^{2}}{\sinh ^{2}(\omega / 2)} \\
\times \ln \left(\frac{e^{q^{2} / 16 \pi+\beta g n_{0}-\omega / 2+\pi \omega^{2} / q^{2}}-e^{-\omega}}{e^{q^{2} / 16 \pi+\beta g n_{0}-\omega / 2+\pi \omega^{2} / q^{2}}-1}\right)^{2}
\end{gathered}
$$

has been calculated before in Ref. 64 for the temperatures above the condensation temperature, the condensate-assisted spin-drag rate,

$$
\begin{aligned}
& \frac{\hbar \beta}{\tau_{12} n \Lambda a_{\uparrow \downarrow}^{2}}=\frac{64}{3(2 \pi)^{3}} \frac{n_{0}}{n} \frac{a}{\Lambda} \int_{0}^{\infty} d p_{1} d p_{3} p_{1} p_{3}^{3} \\
& \quad \times\left(1+\frac{1}{e^{\left(p_{1}^{2}+p_{3}^{2}\right) / 4 \pi+2 \beta g n_{0}}-1}\right) \\
& \quad \times \frac{1}{e^{p_{1}^{2} / 4 \pi+\beta g n_{0}}-1} \frac{1}{e^{p_{3}^{2} / 4 \pi+\beta g n_{0}}-1} \Theta\left(\frac{p_{1} p_{3}}{2 \pi}-\beta g n_{0}\right),
\end{aligned}
$$

where $\Theta$ denotes the Heaviside step function, has not been investigated before. As we can expect, the thermal spin-drag rate $1 / \tau_{22}$ dominates at high temperatures, while the condensate-assisted spin-drag rate $1 / \tau_{12}$ is more important at low temperatures (see Fig. 4). Even though in our approximation the thermal spin-drag rate has a maximum at the critical temperature, a more careful calculation leads to its suppression due to critical fluctuations 65. in a very narrow temperature window around $T_{c}$. Therefore, strictly speaking, $1 / \tau_{12}$ and $1 / \tau_{22}$ vanish at both $T=0$ and $T=T_{c}$.

\section{HYDRODYNAMIC THEORY FOR A BALANCED BINARY MIXTURE}

The goal of this section is to derive a set of hydrodynamic equations for a balanced mixture of two components, where each of the components has a superfluid part and a normal part. Note that the following discussion is, in principle, not limited to weakly interacting Bose gases, since it is only based on conservation laws. However, we do not discuss various dissipative terms such as the thermal diffusivity or the (second) viscosity [17, 60, 66, 67, with the exception of the spin-drag term. Furthermore, in the previous section we have concluded that the spindrag term only relaxes the noncondensate spin currents, whereas in what follows we discuss the hydrodynamics in terms of superfluid and nonsuperfluid (normal) currents. Therefore, we posit that the nonsuperfluid spin currents relax in exactly the same manner as the noncondensate spin currents. This is an approximation, which is only valid in weakly interacting systems, where the condensate and the superfluid are very similar objects.

In the equilibrium situation, the particle densities of the two components are identical for both the superfluid $\left(n_{\uparrow}^{\mathrm{sf}}=n_{\downarrow}^{\mathrm{sf}} \equiv n^{\mathrm{sf}}\right)$ and the normal fluid $\left(n_{\uparrow}^{\mathrm{nf}}=n_{\downarrow}^{\mathrm{nf}} \equiv n^{\mathrm{nf}}\right)$, and therefore the total densities of each species are identical, too $\left(n_{\uparrow}=n_{\uparrow}^{\mathrm{sf}}+n_{\uparrow}^{\mathrm{nf}}=n_{\downarrow}\right)$. Moreover, in equilibrium there are no particle currents $\boldsymbol{j}_{\uparrow}^{\mathrm{nf}}=\boldsymbol{j}_{\downarrow}^{\mathrm{nf}}=\boldsymbol{j}_{\uparrow}^{\mathrm{sf}}=\boldsymbol{j}_{\downarrow}^{\mathrm{sf}}$, as all the velocities vanish: $\boldsymbol{v}_{\uparrow}^{\mathrm{nf}}=\boldsymbol{v}_{\downarrow}^{\mathrm{nf}}=\boldsymbol{v}_{\uparrow}^{\mathrm{sf}}=\boldsymbol{v}_{\downarrow}^{\mathrm{sf}}$, where the normal current of the $\uparrow$ component is defined as $\boldsymbol{j}_{\uparrow}^{\mathrm{nf}}=n_{\uparrow}^{\mathrm{nf}} \boldsymbol{v}_{\uparrow}^{\mathrm{nf}}$, and the other currents are defined similarly. The current of each of the components is the sum of the superfluid and normal currents: $\boldsymbol{j}_{\uparrow}=\boldsymbol{j}_{\uparrow}^{\mathrm{sf}}+\boldsymbol{j}_{\uparrow}^{\mathrm{nf}}$.

In the nonequilibrium situation, however, both the velocities and the various densities can be nonzero and different from each other. In that case, it is useful to define variables pertaining to the combined motion of the whole gas and contrast them to the variables that describe the relative motion. Therefore,

$$
\boldsymbol{j}_{\text {tot }}^{\mathrm{nf}, \mathrm{sf}}=\boldsymbol{j}_{\uparrow}^{\mathrm{nf}, \mathrm{sf}}+\boldsymbol{j}_{\downarrow}^{\mathrm{nf}, \mathrm{sf}},
$$

are the total normal and superfluid currents of the combined motion, respectively. Adding these two currents up yields the total particle current $\boldsymbol{j}_{\text {tot }}=\boldsymbol{j}_{\text {tot }}^{\text {nf }}+\boldsymbol{j}_{\text {tot }}^{\text {sf }}$. Furthermore, we are now in a position to define the combined velocities such that

$$
\boldsymbol{j}_{\text {tot }}^{\mathrm{nf}} \equiv n_{\text {tot }}^{\mathrm{nf}} \boldsymbol{v}_{\text {tot }}^{\mathrm{nf}}
$$

and

$$
\boldsymbol{j}_{\text {tot }}^{\mathrm{sf}} \equiv n_{\text {tot }}^{\mathrm{sf}} \boldsymbol{v}_{\text {tot }}^{\mathrm{sf}} .
$$


Note also that thermodynamic functions (e.g., pressure and entropy) are defined for the whole system only, and not for the individual components. Moreover, due to time-reversal invariance, the lowest-order velocity corrections to thermodynamic functions are quadratic 66] and therefore do not enter linear equations. For example, the Gibbs-Duhem relation reads

$$
n_{\uparrow} d \mu_{\uparrow}+n_{\downarrow} d \mu_{\downarrow}+s d T=d p
$$

even in the case of nonzero velocities. Hence, we obtain the following linearized hydrodynamic equations for the combined (or in-phase) motion of the whole gas:

$$
\begin{aligned}
\partial_{t} n_{\mathrm{tot}}+\boldsymbol{\nabla} \cdot \boldsymbol{j}_{\mathrm{tot}} & =0 \\
\partial_{t}\left(n_{\mathrm{tot}} s\right)+n_{\mathrm{tot}} s \boldsymbol{\nabla} \cdot \boldsymbol{v}_{\mathrm{tot}}^{\mathrm{nf}} & =0 \\
m \partial_{t} \boldsymbol{j}_{\mathrm{tot}}+\boldsymbol{\nabla} p & =0 \\
m \partial_{t} \boldsymbol{v}_{\mathrm{tot}}^{\mathrm{sf}}+\boldsymbol{\nabla} \mu_{\mathrm{tot}} & =0
\end{aligned}
$$

These simple equations express particle conservation, entropy conservation, Newton's second law, and the Josephson relation, respectively. Note that the terms "in-phase" and "out-of-phase" in this paper refer to the motion of the two spin components, as opposed to the normal and the superfluid component, where the latter meaning is common in the literature concerning liquid helium.

We now turn our attention to the relative motion of the up and down particles. To that end, we define the normal and superfluid spin densities

$$
\Delta n^{\mathrm{sf}, \mathrm{nf}}=n_{\uparrow}^{\mathrm{sf}, \mathrm{nf}}-n_{\downarrow}^{\mathrm{sf}, \mathrm{nf}},
$$

as well as spin currents

$$
\Delta j^{\mathrm{sf}, \mathrm{nf}}=j_{\uparrow}^{\mathrm{nf}, \mathrm{sf}}-j_{\downarrow}^{\mathrm{nf}, \mathrm{sf}} .
$$

Using these newly defined quantities, the hydrodynamic spin equations are the following:

$$
\begin{aligned}
\partial_{t} \Delta n^{\mathrm{nf}}+\nabla \cdot \Delta \boldsymbol{j}^{\mathrm{nf}} & =0 \\
\partial_{t} \Delta n^{\mathrm{sf}}+\nabla \cdot \Delta \boldsymbol{j}^{\mathrm{sf}} & =0, \\
\partial_{t} \Delta \boldsymbol{j}^{\mathrm{nf}}+n_{\mathrm{tot}}^{\mathrm{nf}} \nabla \Delta \mu / 2 m & =-\Delta \boldsymbol{j}^{\mathrm{nf}} / \tau_{s d}, \\
\partial_{t} \Delta \boldsymbol{j}^{\mathrm{sf}}+n_{\text {tot }}^{\mathrm{sf}} \nabla \Delta \mu / 2 m & =0 .
\end{aligned}
$$

The four equations above state that both normal and superfluid spin densities are conserved and the normal spin current is driven by a chemical potential difference and relaxes with the spin-drag rate, whereas the superfluid spin current is also driven by a chemical potential difference but does not relax.

By eliminating all the currents and velocities from the equations above and noticing that for the balanced case

$$
\frac{\partial \Delta \mu}{\partial T}=\frac{\partial \Delta \mu}{\partial n}=0
$$

we find that

$$
\begin{aligned}
m \partial_{t}^{2} n_{\text {tot }} & =\nabla^{2} p \\
m \partial_{t}^{2} s & =\frac{n^{\mathrm{sf}}}{n^{\mathrm{nf}}} s^{2} \nabla^{2} T
\end{aligned}
$$

$$
\begin{aligned}
\partial_{t}^{2} \Delta n^{\mathrm{nf}}+\frac{1}{\tau_{s d}} \partial_{t} \Delta n^{\mathrm{nf}}= & \\
& \frac{n^{\mathrm{nf}}}{m} \frac{\partial \Delta \mu}{\partial \Delta n} \nabla^{2}\left(\Delta n^{\mathrm{nf}}+\Delta n^{\mathrm{sf}}\right) \\
\partial_{t}^{2} \Delta n^{\mathrm{sf}}= & \\
& \frac{n^{\mathrm{sf}}}{m} \frac{\partial \Delta \mu}{\partial \Delta n} \nabla^{2}\left(\Delta n^{\mathrm{nf}}+\Delta n^{\mathrm{sf}}\right)
\end{aligned}
$$

We now employ a traveling-wave ansatz for the total density

$$
n_{\text {tot }}=n_{\text {tot }, \mathrm{eq}}+\delta n_{\text {tot }} \exp (-i[\omega t-\boldsymbol{k} \cdot \boldsymbol{x}]),
$$

where $n_{\text {tot,eq }}$ is the total density in the equilibrium, and $\delta n_{\text {tot }}$ is the amplitude of the wave. Proceeding similarly for the other quantities, we obtain the lowest-lying collective modes for the system. Note that in this balanced situation the in-phase collective modes (first and second sounds) are decoupled from the out-of-phase modes (spin modes).

\section{RESULTS}

In this section we present the results of our calculations, most important of which are the sound velocities of the various modes. We discuss both the uniform and the trapped cases. From the total number of hydrodynamic equations, we expect four modes: two in-phase modes and two out-of-phase modes. The two in-phase modes are present in any system where entropy transport is distinct from density transport: They are the first- and the second-sound modes. It is worthwhile to note that the phenomenon of the second sound has been reported not only in superfluid helium, but in other systems as well, including solid helium 68, dielectric crystals [69], and, more recently, a unitary Fermi gas [14]. Moreover, contrary to the case of superfluid helium, second sound in weakly interacting gases is not a pure entropy wave, as discussed below. On the other hand, the out-of-phase modes are similar to spin modes and therefore only occur in systems with several species of particles.

\section{A. Collective modes in a uniform gas}

In the case of no spin drag, all the collective modes of the system have a linear dispersion

$$
\omega=c_{i} k,
$$

where $c_{i}$ are the sound velocities. At zero temperature, the first-sound velocity can be calculated from the expression of pressure in Eq. 44, yielding

$$
c_{1}^{2}=\frac{g_{+} n}{m}=\frac{(1+\gamma) g n}{m} .
$$

Moreover, the second-sound velocity can be calculated in a manner similar to that used in the single-component case (see Ref. [55] for an explicit calculation), the only difference being that, instead of a single phonon gas, we 

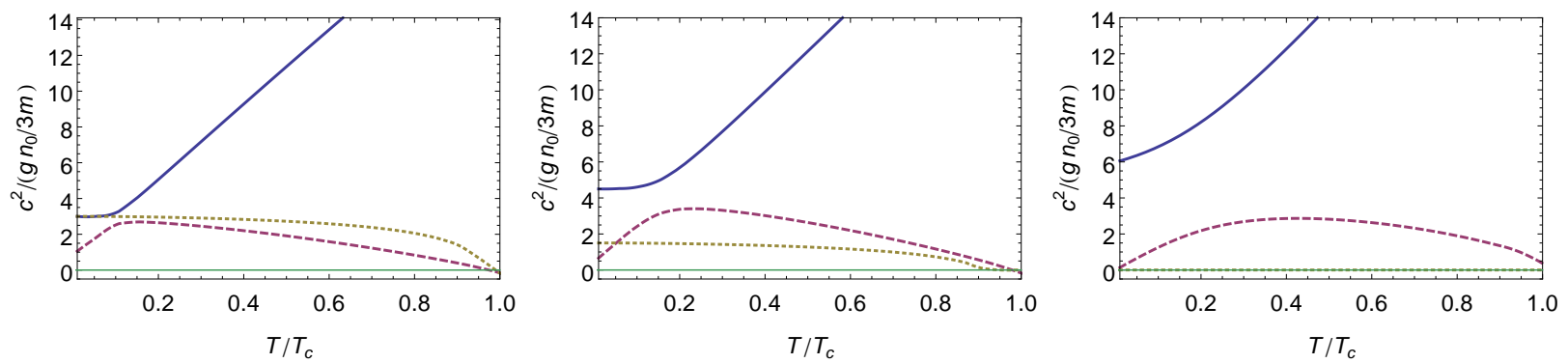

FIG. 5. (Color online) Square of the sound velocities of the two-species balanced Bose gas scaled to the zero-temperature second-sound velocity of a single species. Here $n^{1 / 3} a=0.03$. The inter-component interactions are increasing from left to right: $\gamma=0$ (left), $\gamma=1 / 2$ (center), and $\gamma \lesssim 1$ (right). The latter corresponds to a mixture of hyperfine states of the sodium atom with the density of $10^{21} \mathrm{~m}^{-3}$. The first-sound velocity is depicted by the thick solid line, the second-sound velocity is the dashed line. The spin-sound velocities are the dotted and the thin solid lines.

have to consider a mixture of two noninteracting phonon gases, leading to

$$
c_{2}^{2}=\frac{g n}{3 m} \frac{(1+\gamma)(1-\gamma)^{5 / 2}+(1-\gamma)(1+\gamma)^{5 / 2}}{(1-\gamma)^{5 / 2}+(1+\gamma)^{5 / 2}} .
$$

When the two components are decoupled $(\gamma=0)$, we recover the single-species result $c_{2}^{2}=g n / 3 m$ from the formula above. Furthermore, in the strong-coupling limit $(\gamma \lesssim 1)$, the sound velocity vanishes $\left(c_{2}^{2} \simeq 0\right)$, signaling the demixing transition. When it comes to the spin sounds, one of them always has a zero velocity, whereas the other one has the velocity

$$
c_{s}^{2}=\frac{g_{-} n}{m}=\frac{(1-\gamma) g n}{m},
$$

as can be seen from the difference of chemical potentials in Eq. (22). Note that $c_{1}$ and $c_{s}$ can also be obtained by expanding the energies of the Bogoliubov excitations in Eq. (55) to the lowest order in momentum. Therefore, the first sound and the spin sound can be thought of as both quasiparticles and collective modes at zero temperature.

We present the velocities of the sound modes for different temperatures in Fig. 5. In order to characterize the sound modes, we have calculated the amplitudes of the temperature and density perturbations from the eigenvectors of the linearized system (see Fig. 6). We observe that for the temperatures below the avoided crossing, first sound is mostly a temperature wave. However, above the avoided crossing temperature, first sound is predominantly a density wave. Second sound has comparable contributions from both relative temperature and density deviations for any temperature. Furthermore, second sound is a wave where density and temperature change in phase, while first sound describes an out-ofphase change (the temperature increases with decreasing density), as signified by a minus sign in Fig. 6. When it comes to the spin modes, a zero-frequency mode exists, which corresponds to

$$
\Delta n^{\mathrm{nf}}=-\Delta n^{\mathrm{sf}},
$$

and does not affect the total density of either component. The other spin mode, however, affects the total spin den- sity, while the normal component contributes with the same relative weight as the superfluid component:

$$
\frac{\Delta n^{\mathrm{nf}}}{n^{\mathrm{nf}}}=\frac{\Delta n^{\mathrm{sf}}}{n^{\mathrm{sf}}} .
$$

Upon accounting for spin drag, we find that the zerofrequency modes split into two: one zero-frequency mode and one purely imaginary mode. The purely imaginary mode (cf. Fig. 7) at low momenta is an excitation with only normal density fluctuations, whereas at higher momenta it preserves the total density of every component as the zero-frequency mode: $\Delta n^{\mathrm{nf}}=-\Delta n^{\mathrm{sf}}$. Furthermore, the dispersion of the other spin mode develops a quadratic imaginary part, even though its real velocity $c_{s}$ is almost unaffected by spin drag and the mode is still characterized by Eq. (113). The frequency $\omega$ of the latter mode at long wavelengths can be written as

$$
\omega=c_{s} k-i D k^{2},
$$

where $k$ is the wavenumber and $D$ is the diffusion coefficient. Hence, we call this mode the complex spin mode in Fig. 7 .

Since all the interaction strengths are similar $(\gamma \simeq 1)$ in a sodium gas, when spin drag is absent, both spin modes have zero frequency. In particular, the spin sound has zero velocity at any temperature, $c_{s} \simeq 0$, which is consistent with the zero-temperature result in Eq. 1111. When spin drag is present, the purely imaginary mode has a constant imaginary frequency $\omega=-i / \tau_{s d}$, while the complex mode frequency remains zero, and no imaginary part develops, so $D \simeq 0$ in this case. In a realistic sodium gas with a density of $10^{21} \mathrm{~m}^{-3}$ at half of the critical temperature, the total spin-drag rate is $1 / \tau_{\mathrm{sd}} \simeq 1 \mathrm{kHz}$, while in experiments of trapped sodium gas [48] above $T_{c}$ typical rates are on the order of $0.1 \mathrm{kHz}$. Therefore, it should be experimentally possible to measure the spin-drag rate below the critical temperature by measuring the spin-wave decay time. 

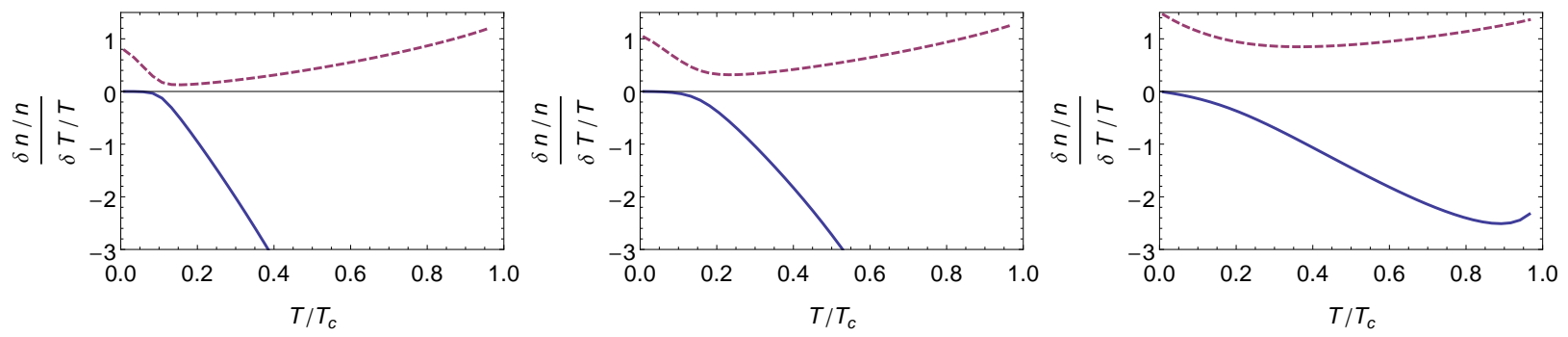

FIG. 6. (Color online) Ratio of density and temperature fluctuations present in first sound (solid line) and second sound (dashed line) for $n^{1 / 3} a=0.03$. Intercomponent interactions are increasing from left to right: $\gamma=0$ (left), $\gamma=1 / 2$ (center), and $\gamma \lesssim 1$ (right). The latter corresponds to a mixture of hyperfine states of the sodium atom with the density of $10^{21} \mathrm{~m}^{-3}$.

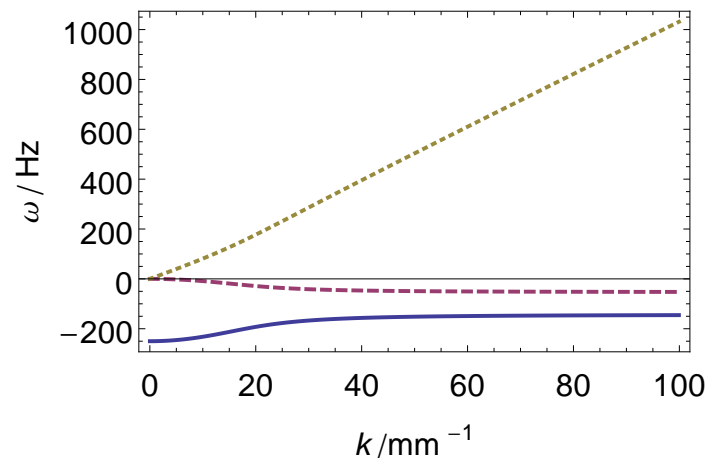

FIG. 7. (Color online) Spin modes in the $n^{1 / 3} a=0.03$ case at $T=T_{c} / 2$ for nonzero interspecies interactions $(\gamma=1 / 2$, $1 / \tau_{s d}=0.25 \mathrm{kHz}$ ). The solid line depicts the imaginary mode, whereas the dashed and dotted lines depict the imaginary and the real parts of the complex spin mode. The horizontal axis corresponds to the physically relevant wavelengths down to about $10 \mu \mathrm{m}$.

\section{B. Gas in a trap}

We now turn our attention to the experimentally relevant trapped case. Here we consider a cylindrically symmetric trap which is highly anisotropic. This trap, where one (axial) direction is very shallow, and the other two (radial) directions are much more strongly confined, puts the gas in the hydrodynamic regime in the axial direction even in the presence of weak interactions. Therefore, we perform the trap average in the radial direction and analyze the propagation of excitations in the axial direction. To that end, we work in the semi-classical approximation (see Ref. [55] for more details). The condensate is treated in the Thomas-Fermi approximation and the excitations are treated in the Hartree-Fock approximation. Therefore, both components are treated in the local-density approximation. We consider the experimentally relevant situation 60] of $2 \cdot 10^{9}$ sodium atoms in a trap with an axial trapping frequency of $2 \pi \times 2 \mathrm{~Hz}$ and a radial trapping frequency of $2 \pi \times 100 \mathrm{~Hz}$, and extract the radial profile at the center of the trap from this calculation. We then perform an average on all the thermodynamic quantities of the hydrodynamic Eqs. (103)- 106). The resulting sound velocities are presented in Fig. 8. At zero temperature, the first-sound velocity in a trap is suppressed by a factor of $\sqrt{2}$ as compared to the uniform case, since the average density in the trap is half of the peak density in the Thomas-Fermi approximation, i.e.,

$$
\left\langle n_{0}\right\rangle=n_{0} / 2
$$

as first pointed out by Zaremba [70]. Since at zero temperature the second-sound velocity is also proportional to the square root of the density, it is suppressed by the same factor of $\sqrt{2}$. This is not explicit for sodium atoms as the second sound at zero temperature vanishes. However, for a nonzero temperature we have not succeeded in finding a similar simple relation between the trapped and uniform gases. Finally, we also present the spin-drag rate dependence on temperature for a trapped system (Fig. 9). The curves are qualitatively similar to the uniform case, even though the rates are decreased by a factor of about 10 .

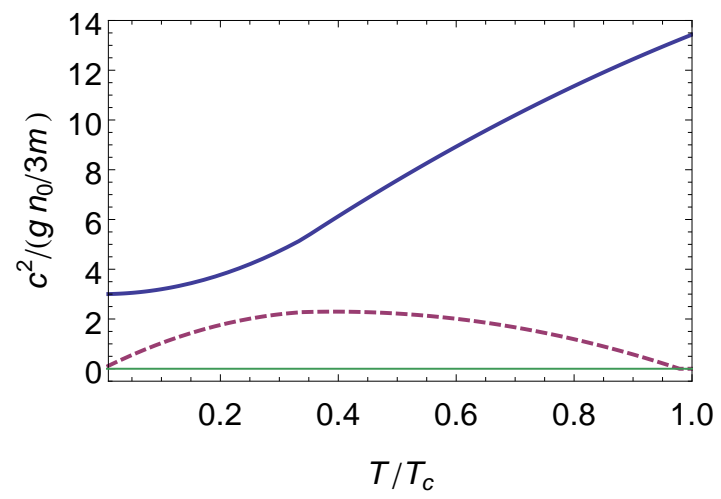

FIG. 8. (Color online) Trap averaged sound velocities normalized to the peak density in the trap with no damping (see text for details).

\section{CONCLUSION}

In summary, we have constructed a hydrodynamic theory of a balanced two-species Bose mixture. We have also calculated the microscopic thermodynamic parame- 


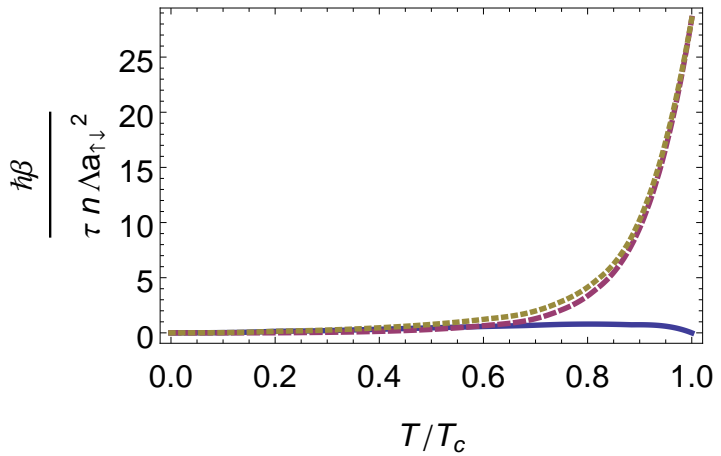

FIG. 9. (Color online) Trap averaged condensate-assisted (solid line), thermal (dashed line), and total (dotted line) spin-drag rates normalized to the peak density in the trap (see text for details).

ters entering that theory using the Popov approximation, obtaining the equation of state on the way. Moreover, we have accounted for the relaxation of the normal current by the spin-drag mechanism, considering the condensatemediated spin-drag term for the first time. Adding these components together we were able to calculate the sound velocities and spin-drag rates for the experimentally accessible system of sodium atoms in a trap. We hope that our analysis will stimulate experimental work on hydrodynamic modes and spin drag in a partially condensed Bose mixture.

\section{ACKNOWLEDGMENTS}

This work was supported by the Stichting voor Fundamenteel Onderzoek der Materie (FOM) and the European Research Council (ERC) and is part of the DITP consortium, a program of the Netherlands Organisation for Scientific Research (NWO) that is funded by the Dutch Ministry of Education, Culture and Science $(\mathrm{OCW})$. It is our pleasure to thank M. Di Liberto, S. Grubinskas, and C. Khripkov for stimulating discussions.
[1] M. H. Anderson, J. R. Ensher, M. R. Matthews, C. E. Wieman, and E. A. Cornell, Science 269, 198 (1995), http://www.sciencemag.org/content/269/5221/198.full.pdf

[2] K. B. Davis, M. O. Mewes, M. R. Andrews, N. J. van Druten, D. S. Durfee, D. M. Kurn, and W. Ketterle, Phys. Rev. Lett. 75, 3969 (1995)

[3] C. C. Bradley, C. A. Sackett, J. J. Tollett, and R. G. Hulet, Phys. Rev. Lett. 75, 1687 (1995)

[4] A. J. Leggett, Rev. Mod. Phys. 73, 307 (2001).

[5] I. Bloch, J. Dalibard, and W. Zwerger, Rev. Mod. Phys. 80, 885 (2008)

[6] S. Stringari, Phys. Rev. Lett. 77, 2360 (1996)

[7] D. S. Jin, J. R. Ensher, M. R. Matthews, C. E. Wieman, and E. A. Cornell, Phys. Rev. Lett. 77, 420 (1996)

[8] M.-O. Mewes, M. R. Andrews, N. J. van Druten, D. M. Kurn, D. S. Durfee, C. G. Townsend, and W. Ketterle, Phys. Rev. Lett. 77, 988 (1996)

[9] N. Bogoljubov, Il Nuovo Cimento 7, 794 (1958).

[10] A. Griffin, Phys. Rev. B 53, 9341 (1996)

[11] J. O. Andersen, Rev. Mod. Phys. 76, 599 (2004)

[12] J. M. Vogels, K. Xu, C. Raman, J. R. Abo-Shaeer, and W. Ketterle, Phys. Rev. Lett. 88, 060402 (2002)

[13] A.-C. Voigt, M. Taglieber, L. Costa, T. Aoki, W. Wieser, T. W. Hänsch, and K. Dieckmann, Phys. Rev. Lett. 102, 020405 (2009)

[14] L. A. Sidorenkov, M. K. Tey, R. Grimm, Y.-H. Hou, L. Pitaevskii, and S. Stringari, Nature 498, 78 (2013).

[15] F. Schreck, L. Khaykovich, K. L. Corwin, G. Ferrari, T. Bourdel, J. Cubizolles, and C. Salomon, Phys. Rev. Lett. 87, 080403 (2001)

[16] W. Colson and A. Fetter, Journal of Low Temperature Physics 33, 231 (1978)

[17] T.-L. Ho and V. Shenoy, Journal of Low Temperature Physics 111, 937 (1998)

[18] R. Graham and D. Walls, Phys. Rev. A 57, 484 (1998).
[19] B. D. Esry and C. H. Greene, Phys. Rev. A 59, 1457 (1999)

[20] D. V. Skryabin, Phys. Rev. A 63, 013602 (2000)

[21] P. Tommasini, E. J. V. de Passos, A. F. R. de Toledo Piza, M. S. Hussein, and E. Timmermans, Phys. Rev. A 67, 023606 (2003).

[22] B. Dalton and S. Ghanbari, Journal of Modern Optics 59, $287 \quad$ (2012), http://dx.doi.org/10.1080/09500340.2011.632100.

[23] M. Abad and A. Recati, The European Physical Journal D 67, 148 (2013), 10.1140/epjd/e2013-40053-2

[24] K. Anoshkin, Z. Wu, and E. Zaremba, Phys. Rev. A 88, 013609 (2013)

[25] M. J. Edmonds, K. L. Lee, and N. P. Proukakis, Phys. Rev. A 91, 011602 (2015).

[26] D. S. Hall, M. R. Matthews, J. R. Ensher, C. E. Wieman, and E. A. Cornell, Phys. Rev. Lett. 81, 1539 (1998).

[27] G. Modugno, M. Modugno, F. Riboli, G. Roati, and M. Inguscio, Phys. Rev. Lett. 89, 190404 (2002)

[28] S. Beattie, S. Moulder, R. J. Fletcher, and Z. Hadzibabic, Phys. Rev. Lett. 110, 025301 (2013)

[29] C. Hamner, Y. Zhang, J. J. Chang, C. Zhang, and P. Engels, Phys. Rev. Lett. 111, 264101 (2013)

[30] C.-H. Zhang and H. A. Fertig, Phys. Rev. A 75, 013601 (2007).

[31] Z.-Q. Yu, Phys. Rev. A 90, 053608 (2014)

[32] T.-L. Ho, Phys. Rev. Lett. 81, 742 (1998)

[33] T. Ohmi and K. Machida, Journal of the Physical Society of Japan 67, 1822 (1998)

[34] D. M. Stamper-Kurn and M. Ueda, Rev. Mod. Phys. 85, 1191 (2013)

[35] M.-S. Chang, Q. Qin, W. Zhang, L. You, and M. S. Chapman, Nat Phys 1, 111 (2005)

[36] L. E. Sadler, J. M. Higbie, S. R. Leslie, M. Vengalattore, and D. M. Stamper-Kurn, Nature 443, 312 (2006). 
[37] M. W. Ray, E. Ruokokoski, S. Kandel, M. Mottonen, and D. S. Hall, Nature 505, 657 (2014), letter.

[38] U. Al Khawaja and H. Stoof, Nature 411, 918 (2001).

[39] H. T. C. Stoof, E. Vliegen, and U. Al Khawaja, Phys. Rev. Lett. 87, 120407 (2001)

[40] H. Pu, S. Raghavan, and N. P. Bigelow, Phys. Rev. A 63, 063603 (2001).

[41] L. S. Leslie, A. Hansen, K. C. Wright, B. M. Deutsch, and N. P. Bigelow, Phys. Rev. Lett. 103, 250401 (2009)

[42] J. Armaitis, H. T. C. Stoof, and R. A. Duine, Phys. Rev. Lett. 110, 260404 (2013)

[43] J.-y. Choi, S. Kang, S. W. Seo, W. J. Kwon, and Y.-i. Shin, Phys. Rev. Lett. 111, 245301 (2013)

[44] Y. Kawaguchi, M. Nitta, and M. Ueda, Phys. Rev. Lett. 100, 180403 (2008)

[45] M. Polini and G. Vignale, Phys. Rev. Lett. 98, 266403 (2007)

[46] R. A. Duine and H. T. C. Stoof, Phys. Rev. Lett. 103, $170401(2009)$

[47] A. Sommer, M. Ku, G. Roati, and M. W. Zwierlein, Nature 472, 201 (2011).

[48] S. B. Koller, A. Groot, P. C. Bons, R. A. Duine, H. T. C. Stoof, and P. van der Straten, ArXiv e-prints (2012), arXiv:1204.6143 [cond-mat.quant-gas]

[49] S. O. Demokritov, V. E. Demidov, O. Dzyapko, G. A. Melkov, A. A. Serga, B. Hillebrands, and A. N. Slavin, Nature 443, 430 (2006).

[50] R. E. Troncoso and A. S. Núñez, Annals of Physics 346, 182 (2014)

[51] W. H. Bassichis, Phys. Rev. 134, A543 (1964)

[52] D. M. Larsen, Annals of Physics 24, 89 (1963)

[53] G. M. Falco, A. Pelster, and R. Graham, Phys. Rev. A 75, 063619 (2007).

[54] H. T. C. Stoof, K. B. Gubbels, and D. B. M. Dickerscheid, Ultracold Quantum Fields (Springer, Dordrecht, 2009).

[55] C. J. Pethick and H. Smith, Bose-Einstein Condensation in Dilute Gases; 2nd ed. (Cambridge Univ. Press, Cam- bridge, 2008).

[56] T. D. Lee and C. N. Yang, Phys. Rev. 105, 1119 (1957)

[57] T. D. Lee, K. Huang, and C. N. Yang, Phys. Rev. 106, 1135 (1957).

[58] In the grand-canonical ensemble thermodynamic quantities are obtained by considering the grand potential $\Omega$ that explicitly depends on the chemical potentials. In that case, the density is $n_{\sigma}=-\partial \Omega /\left.\partial \mu_{\sigma}\right|_{T}$, and the entropy per volume is $S / V=-\partial \Omega /\left.\partial T\right|_{\mu_{\sigma}}$. We have checked that the quantities obtained that way match the ones given in the main text. The said calculation was not presented here in detail, since the relevant expressions (including the dispersions of the quasiparticles) become rather cumbersome.

[59] S. Knoop, T. Schuster, R. Scelle, A. Trautmann, J. Appmeier, M. K. Oberthaler, E. Tiesinga, and E. Tiemann, Phys. Rev. A 83, 042704 (2011).

[60] R. Meppelink, R. van Rooij, J. M. Vogels, and P. van der Straten, Phys. Rev. Lett. 103, 095301 (2009)

[61] S. Floerchinger and C. Wetterich, Phys. Rev. A 79, 063602 (2009)

[62] T. Nikuni, E. Zaremba, and A. Griffin, Phys. Rev. Lett. 83, 10 (1999).

[63] E. Zaremba, T. Nikuni, and A. Griffin, Journal of Low Temperature Physics 116, 277 (1999)

[64] H. van Driel, R. Duine, and H. Stoof, Phys. Rev. Lett. 105, 155301 (2010).

[65] R. Kittinaradorn, R. A. Duine, and H. T. C. Stoof, New Journal of Physics 14, 055007 (2012)

[66] I. M. Khalatnikov, An Introduction To The Theory of Superfluidity (Westview Press, 2000).

[67] T. Nikuni and A. Griffin, Phys. Rev. A 63, 033608 (2001).

[68] C. C. Ackerman, B. Bertman, H. A. Fairbank, and R. A. Guyer, Phys. Rev. Lett. 16, 789 (1966)

[69] T. F. McNelly, S. J. Rogers, D. J. Channin, R. J. Rollefson, W. M. Goubau, G. E. Schmidt, J. A. Krumhansl, and R. O. Pohl, Phys. Rev. Lett. 24, 100 (1970).

[70] E. Zaremba, Phys. Rev. A 57, 518 (1998) 